\title{
Nonlinear solitary waves for the nondestructive evaluation of concrete
}

\author{
Piervincenzo Rizzo, Ph.D., Professor \\ Laboratory for Nondestructive Evaluation and Structural Health Monitoring Studies \\ Department of Civil and Environmental Engineering \\ University of Pittsburgh, Pittsburgh, PA, USA
}

\begin{abstract}
In the last two decades it has been demonstrated that highly nonlinear solitary waves (HNSWs) propagating in chains of granular particles can be used in many physics and engineering applications, including acoustic lenses, impurity detectors, and nondestructive evaluation (NDE). HNSWs are compact nondispersive waves that propagate in nonlinear medium such as 1D chains of spherical particles. In this talk the application of HNSWs for the nondestructive evaluation (NDE) of concrete material is presented. The non-invasive assessment of cement and concrete strength are discussed to describe how the solitary waves can be used to monitor curing, estimate the strength of concrete, or detect the presence of excessive water.
\end{abstract}

